Cipango Cahiers d'études japonaises

19 | 2012

Le Japon et le fait colonial II

\title{
Impérialiste contre voyou
}

Le Japon, la Corée du Nord et la question coloniale après 1945

Imperialist vs Rogue. Japan, North Korea and the Colonial Question since 1945

\section{Adrien Carbonnet}

\section{(2) OpenEdition}

Journals

Édition électronique

URL : https://journals.openedition.org/cipango/1709

DOI : 10.4000/cipango.1709

ISSN : 2260-7706

Éditeur

INALCO

Édition imprimée

Date de publication : 30 octobre 2012

Pagination : 149-171

ISBN : 978-2-85831-204-7

ISSN : $1164-5857$

Référence électronique

Adrien Carbonnet, «Impérialiste contre voyou », Cipango [En ligne], 19 | 2012, mis en ligne le 15 mai 2014 , consulté le 30 juin 2021. URL : http://journals.openedition.org/cipango/1709 ; DOI : https:// doi.org/10.4000/cipango.1709

Ce document a été généré automatiquement le 30 juin 2021.

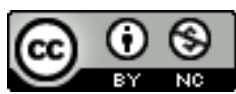

Cipango est mis à disposition selon les termes de la Licence Creative Commons Attribution - Pas d'Utilisation Commerciale 4.0 International. 


\section{Impérialiste contre voyou}

Le Japon, la Corée du Nord et la question coloniale après 1945

Imperialist vs Rogue. Japan, North Korea and the Colonial Question since 1945

\section{Adrien Carbonnet}

1 Premier pays auquel le Japon imposa un traité inégal ${ }^{1}$, enjeu des guerres sino-japonaise (1894-1895) et russo-japonaise (1904-1905), protectorat en 1905 puis annexée cinq ans plus tard, la Corée occupa une place centrale dans la politique étrangère du Japon de Meiji (1868-1912). Restée sous domination japonaise pendant plus de quarante ans, la péninsule ne recouvra son indépendance qu'après la capitulation du Japon, en 1945, avant de devenir un des théâtres de l'affrontement Est-Ouest. Le 8 septembre 1951, en pleine guerre de Corée (1950-1953), le Japon, de son côté, signait un traité de sécurité avec les États-Unis, renforçant encore un peu plus son point d'ancrage au bloc occidental. Des deux républiques coréennes, c'est donc presque naturellement celle au Sud, basculée à l'Ouest, que Tōkyō choisit pour instaurer des relations officielles, après de longues et difficiles négociations qui aboutirent, le 22 juin 1965, à la conclusion du Traité général entre le Japon et la République de Corée (Nihonkoku to daikanminkoku to no aida no kihon kankei no jōyaku 日本国と大韓民国との間の基本関係の条約).

Désormais engagé dans une relation qui se voulait exclusive dans un contexte de guerre froide, conforté dans sa réserve par le comportement délinquant de P'yŏngyang² Tōkyō ne normalisa pas - et n'a d'ailleurs toujours pas normalisé - ses relations avec l'État au nord $\mathrm{du} 38^{\mathrm{e}}$ parallèle. Ce vide institutionnel fut toutefois en partie comblé par l'établissement de contacts - économiques, politiques et intellectuels - entre les deux pays. Sur l'échiquier politique japonais, c'est le Parti communiste (PCJ) - dont le premier programme de 1922 condamnait l'annexion de la Corée par le Japon impérialiste et engageait le Parti à tout mettre en œuvre pour la libération de la péninsule - qui fut, à la fin de la Seconde Guerre mondiale, le premier interlocuteur de P'yŏngyang, jusqu'à ce que ce rôle incombe au Parti socialiste (PSJ), puis à certaines personnes influentes du Parti libéral-démocrate (PLD). Les autorités nord-coréennes pensèrent trouver au sein du parti au pouvoir depuis 1955 des hommes de poids capables de mobiliser la métropole en faveur de son ancienne colonie d'outre-mer. 
3 La question coloniale qui nous intéresse revêt des aspects multiples : elle est tour à tour objet d'étude pour les historiens, passé à reconnaître, à nier voire à instrumentaliser pour les hommes politiques, et vécu des peuples. Elle implique une posture différente selon la catégorie d'acteur que l'on considère, de la quête de la vérité à la poursuite de l'intérêt national en passant par la volonté de reconnaissance et de justice. Ces différents niveaux d'analyse, mus par leur propre logique, mais s'entremêlant souvent, rendent malaisée une approche globale de la question. Aussi, la présente contribution s'en tiendra à deux axes de réflexion: la question coloniale comme enjeu des négociations nippo-nord-coréennes ; la place occupée, depuis ces dix dernières années, par cette même question au sein de la sphère publique au Japon.

\section{Un passé à négocier}

\section{« Régler le passé »}

4 Que l'on soit au Japon ou en Corée, il est une expression qui revient fréquemment lorsque l'on se penche sur la question coloniale: le "règlement du passé » ou « liquidation du passé » (kako no seisan 過去の清算 / kwagŏ ch’ŏngsan 과거청산). Pour deux des trois figures évoquées plus haut - l'historien et le peuple - l'expression semble ne pas avoir réellement de sens. La communauté des historiens peut-elle en effet considérer le passé comme "réglé » lorsqu'un consensus sur les réalités de la colonisation est trouvé ? L'équilibre trouvé serait nécessairement précaire, bousculé par de nouvelles recherches historiques, remis en cause par de nouveaux débats. La question est-elle réglée lorsque sont rétablis des échanges entre les deux territoires? Lorsque la majorité d'un peuple autrefois colonisé n'éprouve plus d'animosité envers l'ancien colonisateur? Les critères de jugement semblent se dérober un peu plus encore lorsque l'on situe la réflexion au niveau du peuple. Le "règlement du passé » semble n'avoir en réalité de sens qu'au niveau diplomatique; il inclut alors le plus souvent deux volets : des excuses orales et/ou écrites par un responsable politique - le plus souvent un chef d'État ou de gouvernement - et le versement d'indemnités matérielles. La notion d'excuses n'est à son tour pas dénuée d'ambiguïtés.

5 La notion d'excuses n'est à son tour pas dénuée d'ambiguïtés. Qu'est-ce que présenter des excuses pour un État ${ }^{3}$ ? Est-ce s'agenouiller, tel Willy Brandt en 1970 devant le mémorial du soulèvement du ghetto de Varsovie, acte qui nous paraît hautement symbolique aujourd'hui, mais qui ne fut pas exempt de critiques en son temps ${ }^{4}$ ? Est-ce affirmer que l'État s'est « trompé de politique » et reconnaître les « faits incontestables de l'histoire», comme le fit le Premier ministre Murayama Tomi.ichi 村山富市 lors de la commémoration du cinquantième anniversaire de la fin de la Seconde Guerre mondiale, le 15 août 1995 ?

Pendant une période de son passé pas si éloignée de nous, le Japon s'est fourvoyé dans sa politique et a pris le chemin de la guerre, mettant en danger la vie du peuple japonais. Par son agression et sa domination coloniale, le Japon a causé d'importants dommages et souffrances aux peuples de nombreuses nations, en particulier celles d'Asie. Pour éviter toute erreur à l'avenir, je reconnais avec humilité les faits incontestables de l'histoire, et tiens ici à exprimer à nouveau mes profonds remords et mes excuses sincères. 
わが国は、遠くない過去の一時期、国策を誤り、戦争への道を步んで国民を存亡 の危機に陥机、植民地支配と侵略によって、多くの国々、とりわけアジア諸国の 人々に対して多大の損害と苦痛を与えました。私は、未来に誤ち無からしめんと するが故に、疑うべくもないこの歴史の事実を謙虚に受け止め、ここにあらため て痛切な反省の意を表し、心からのお詫びの気持ちを表明いたします5。

Cet extrait nous montre le caractère fondamentalement "ouvert» des excuses formulées, que ce soit au niveau de leurs destinataires (les peuples de nombreuses nations), de ce qui les motive (l'agression et la domination coloniale) ou des conséquences (d'importants dommages et souffrances). Il s'agit de faire consensus, de trouver un dénominateur commun qui permettrait de se mettre à l'abri des controverses d'historiens et des querelles politiques. Qui contesterait aujourd'hui que «par son agression et sa domination coloniale, le Japon a causé d'importants dommages et souffrances aux peuples de nombreuses nations, en particulier celles d'Asie " ? Ce sont, en quelque sorte, des excuses de «bon sens", et si quelque chose devait susciter le débat, ce ne serait pas tant le contenu des excuses que le fait de les présenter. Notons également qu'à la différence de l'acception traditionnelle selon laquelle excuse et pardon vont de pair (on présente des excuses en espérant de l'autre qu'il les accepte), le geste de l'État fautif n'est presque jamais suivi du pardon de l'État offensé. Qu'importe d'ailleurs, puisque les excuses ne seraient pas tant adressées à la victime qu'à la société internationale et viseraient à recouvrer un honneur perdu, à s'affirmer comme un acteur responsable et capable de faire face à l'Histoire.

\section{Entre diplomatie des parlementaires et diplomatie d'État}

Dans le cas de la Corée du Nord, il faut remonter au 30 mars 1989. Interpellé à la commission du Budget de la Chambre des Conseillers par le socialiste Murayama Tomi.ichi, Takeshita Noboru 竹下登, le Premier ministre de l'époque, déclara être conscient des «nombreuses souffrances et torts » (tadai no kutsū to songai 多大の苦痛と 損害) que le Japon et son peuple avaient causés par le passé à ses voisins. Il exprima à «toutes les personnes de la péninsule coréenne »-sous-entendues celles au nord du $38^{\mathrm{e}}$ parallèle comprises - ses « profonds regrets et remords 》 (fukai hansei to ikan 深い 反 省と遺憾) avant d'appeler au dialogue entre les deux gouvernements. Cet acte revêtait une forte portée symbolique, car c'était la première fois qu'un chef de gouvernement japonais utilisait le nom officiel de « République populaire démocratique de Corée » à la place de "Corée du Nord", signe d'une reconnaissance de l'État établi au nord du $38^{\mathrm{e}}$ parallèle ${ }^{6}$.

8 À la suite de cette déclaration, une délégation de parlementaires présidée par l'ancien vice-Premier Ministre Kanemaru Shin 金丸信, membre influent du PLD, et Tanabe Makoto 田邊誠, alors vice-secrétaire général du PSJ - deux figures importantes de la vie politique japonaise qui, quoique d'affiliation partisane différente, entretenaient des liens privilégiés avec la Corée du Nord-, se rendit à P'yŏngyang du 24 au 28 septembre 1990. La visite déboucha sur la signature de la Déclaration commune des trois Partis ${ }^{7}$ (Santō kyōdōsengen 三党共同宣言) qui prévoyait que les trois formations politiques agissent «fermement [auprès de leur gouvernement respectif] afin que débutent dans le courant du mois de novembre [1990] des négociations intergouvernementales visant à établir des relations diplomatiques et à régler les différentes questions pendantes ». Si cette visite fut dans son ensemble plutôt bien 
accueillie par la presse, la partie de la déclaration reproduite ci-dessous suscita un tollé, y compris au sein du PLD.

Les trois Partis reconnaissent que le Japon doit présenter des excuses officielles et dédommager convenablement la République populaire démocratique de Corée pour les malheurs et les torts causés par le passé au peuple coréen pendant trente-six ans, ainsi que pour les pertes qu'il lui a infligées durant les quarante-cinq années qui ont suivi la fin de la guerre.

三党は、過去に日本が 36 年間朝鮮人民に与えた大きな不幸と炎難、戦後 45 年 間朝鮮人民が受けた損失について、朝鮮民主主義人民共和国に対し、公式的に謝 罪を行い十分に償うべきであると認める。

9 C'est la dernière partie de la phrase "ainsi que pour les pertes qu'il lui a infligées durant les quarante-cinq années qui ont suivi la fin de la guerre » qui posa problème. La domination du Japon sur la Corée ayant pris fin avec la capitulation japonaise, il était inacceptable, pour le ministère des Affaires étrangères et le gouvernement japonais, d'assumer une quelconque responsabilité pour les années après 1945. Le politologue Ōtake Hideo impute cette maladresse à la personnalité de Kanemaru Shin qui, « en plus d'être un novice dans la diplomatie, sans aucune expérience des négociations internationales, se laissait facilement prendre par les sentiments » ${ }^{8}$. Aussi, «touché par les flatteries et l'éloquence dont fit preuve Kim Il-sŏng 김일성 金日成, par l'accueil fastueux qui lui avait été réservé et par la promesse de son hôte de libérer [deux marins japonais retenus prisonniers en Corée du Nord], il signa la déclaration commune, une copie conforme des positions nord-coréennes » (ibid.).

10 Les diplomates prirent le relais le 30 janvier 1991, et huit réunions eurent lieu jusqu'en novembre 1992. La question coloniale, à cheval sur trois des quatre thèmes de discussion définis lors de réunions préliminaires ${ }^{9}$, posa particulièrement problème. La Corée du Nord considérait en effet qu'elle avait été en guerre avec le Japon et qu'en 1945 elle était sortie victorieuse de ce combat, d'où sa demande de " réparations » au vaincu japonais. P'yŏngyang sut également tirer profit de la brèche ouverte par Kanemaru et Tanabe, et exigea des indemnités pour les quarante-cinq années après 1945. De son côté, la partie japonaise soutenait que le Japon et la Corée n'avaient jamais été en guerre, et qu'il ne pourrait être, par conséquent, question de réparations ou d'indemnités, à fortiori pour les années suivant la capitulation. Le Nord mit fin, en 1992, à cette première série de pourparlers et les trois autres réunions qui eurent lieu d'avril à octobre 2000 ne permirent pas de régler les questions relatives au passé. Il fallut attendre la rencontre au sommet du 17 septembre 2002 entre Koizumi Jun.ichirō et Kim Chŏng-il 김정 일 金正日 pour que la situation se débloque. Cette première visite d'un Premier ministre japonais en Corée du Nord - préparée dans le secret et annoncée tardivement au partenaire américain- déboucha sur la reconnaissance des enlèvements de Japonais (voir infra) et sur la signature de la Déclaration de P'yŏngyang (nitchō pyonyan sengen 日朝平塞 宣言). Ce texte, qui prévoyait l'ouverture de négociations pour normaliser les relations entre les deux États (point 1), engage la Corée du Nord sur les questions de sécurité (point 4) et sur la sécurité des nationaux japonais (point 3). Quant à la question coloniale, le point 2 dispose que :

La partie japonaise, reconnaissant avec humilité les faits de l'histoire selon lesquels le Japon a causé au peuple coréen de nombreux dommages et souffrances par la 
domination coloniale qu'il a exercée dans le passé, a manifesté un profond remord et a exprimé ses excuses ${ }^{10}$ sincères.

日本側は、過去の植民地支配によって、朝鮮の人々に多大の損害と苦痛を与えた という歴史の事実を謙虚に受け止め、痛切な反省と心からのお詫びの気持ちを表 明した。

11 Le texte prévoyait également la mise en place d'une " coopération économique » (keizai kyōryoku 経済協力) constituée de dons, de prêts à long terme et à intérêt réduit, ainsi que d'aides humanitaires. Cette manière de procéder n'est pas sans rappeler la méthode qui fut finalement retenue pour la Corée du Sud ${ }^{11}$. Le diplomate japonais en charge de la rencontre justifia d'ailleurs ce choix par le fait qu'il lui était impossible d'avoir recours à un mode de règlement différent de celui adopté pour le Sud quelques dizaines d'années auparavant ${ }^{12}$. Si les pourparlers engagés à la suite de cette déclaration butèrent sur la question des enlèvements de Japonais et celle du nucléaire, sa signature semble avoir bel et bien "réglé " la question coloniale. Et le fait que la Corée du Nord se mit à réclamer à nouveau, notamment lors de la treizième session des pourparlers en février 2006, des «indemnités » - semblant ainsi remettre en question la «coopération économique » prévue par la déclaration de P'yŏngyang - doit être interprété comme une stratégie visant à déstabiliser la partie japonaise plutôt que comme une réelle remise en cause de l'accord. Il est d'ailleurs fort peu probable que le Japon revienne un jour sur cette forme de dédommagement.

\section{Question coloniale et sphère publique}

\section{Un intérêt secondaire}

Quelle que fût la place exacte du «Grand dirigeant » dans la résistance - il joua très certainement un rôle moindre que celui que lui prêtent les hagiographies officielles ${ }^{13}$ le combat contre les Japonais, gravé dans les statuts du Parti du travail de Corée depuis sa création, légitima la place de Kim Il-sŏng à la tête du Parti et de l'État jusqu'à sa mort en 1994. Cette lutte antijaponaise, élément fondamental du culte de la personnalité, s'est accaparée l'espace public nord-coréen dans sa version glorifiée : elle est intrigue dans le théâtre et la littérature, elle est projetée sur de vastes panneaux explicatifs dans les musées, elle façonne enfin l'architecture de la capitale au centre de laquelle, sur la colline Mansu, se dresse une immense statue en bronze de Kim Il-sŏng flanquée d'un monument dédié à la lutte contre l'occupant.

Cette lutte sacrée est assimilable à une clef de voûte soutenant tout l'ensemble. À l'instar du rôle d'Emmanuel Goldstein, le personnage de 1984, «traître fondamental, le premier profanateur de la pureté du Parti [dont] tous les crimes subséquents contre le Parti, trahisons, actes de sabotage, hérésies, déviations, jaillissent directement de son enseignement $~^{14}$, dont le visage apparaît chaque jour sur les écrans d'Océania durant les Deux Minutes de la Haine, le régime nord-coréen se doit de maintenir en vie la figure du Japon impérialiste pour assurer sa propre survie. Ces considérations peuvent expliquer en partie la persistance de la Corée du Nord à réclamer au Japon des réparations puis des indemnités pour son passé colonial. Y renoncer serait fléchir à nouveau devant l'ennemi, au risque d'ébranler le socle du régime, de faire vaciller l'édifice dans son ensemble. En proie à l'aggravation de sa situation économique dans les années 1990, P'yŏngyang dut pourtant assouplir sa position : la somme que verserait 
le Japon pour régler son passé - même sous la forme édulcorée et finalement un peu honteuse de "coopération économique »- constituerait un afflux de devises bienvenu et sauverait les apparences ${ }^{15}$.

L'omniprésence nécessaire de la thématique coloniale en Corée du Nord contraste avec l'intérêt relativement limité pour cette même question au Japon. Le tableau ci-dessous présente une série d'enquêtes d'opinion concernant la diplomatie (gaikō ni kansuru yoron chōsa 外交に関する世論調査) conduites en 2000, puis chaque année entre 2002-2011 par le bureau du gouvernement (naikakufu 内閣府). Il a été demandé aux Japonais interrogés quels étaient les sujets relatifs à la Corée du Nord qui les intéressaient (kanshin o motsu 関心を持つ). Ce sont les cinq premiers items cités par les sondés qui ont été pris en compte.

Les enquêtes d'opinion sont des instruments à manier avec précaution dans la mesure où la réponse d'un chercheur ayant consacré sa vie à la question coloniale compte autant que celle de quelqu'un qui, avant d'être sollicité pour répondre au sondage, ignorait que la Corée avait été une colonie. Aussi, affirmer au regard des résultats d'un sondage que la colonisation n'intéresse pas les Japonais reviendrait à nier les milliers de pages écrites par les universitaires japonais sur le sujet ${ }^{16}$. Les enquêtes d'opinion n'en ont pas moins le mérite de donner une photographie «à chaud »-certes pleinement lisible que si resituée dans son contexte - et peuvent décrire une tendance lorsqu'elles s'inscrivent dans la durée. Les sondages compilés et présentés ci-dessous révèlent d'abord que la question coloniale n'est proposée dans les choix de réponse qu'à travers le prisme $d u$ "règlement du passé ${ }^{17}$, et non pas sous des libellés tels que " colonie " (shokuminchi 植民地), 《domination coloniale» (shokuminchi shihai 植民地支配) ou même « histoire des relations nippo-coréennes » (nitchō kankei-shi 日朝関係史). Ils nous montrent ensuite que la part des Japonais avouant s'intéresser au poste « règlement du passé » n'a jamais dépassé le quart de la population interrogée et oscille entre 24,6 \% (2000) et $16,9 \%$ (2004). Cette thématique arrive loin derrière celles des enlèvements de Japonais (au-dessus des $80 \%$ depuis 2002 avec un pic à plus de $90 \%$ en 2003), des questions sécuritaires (quel que soit le poste concerné : arme nucléaire, missiles, embarcations suspectes opérant dans la clandestinité, trafics illégaux) et même derrière les « relations intercoréennes » (entre $20 \%$ et $30 \%$ depuis 2002).

Ces sondages soulignent également une corrélation entre les sujets d'actualité fortement médiatisés et l'intérêt que portent les personnes interrogées à ces sujets. Ainsi, alors qu'en 2000 - année de la rencontre (relayée par les médias japonais) entre le président sud-coréen et le dirigeant nord-coréen - 47,9\% des Japonais se disaient intéressés par les relations intercoréennes, cette part ne représentait plus que 27,7\% deux ans plus tard. Il en va de même de l'intérêt des Japonais pour l'arme nucléaire nord-coréenne qui atteint, après le premier essai (octobre 2006) et le deuxième essai (mai 2009), des proportions particulièrement importantes : respectivement 79,5\% et $76,8 \%$. 


\section{Sujets d'intérêt des Japonais pour la Corée du Nord ${ }^{18}$}

\begin{tabular}{|c|c|c|c|c|c|c|c|c|c|c|c|}
\hline THÈMES / ANNÉES & 2000 & 2002 & 2003 & 2004 & 2005 & 2006 & 2007 & 2008 & 2009 & 2010 & 2011 \\
\hline ENLÈVEMENTS & 68,6 & 83,4 & 90,1 & 88,3 & 87,6 & 86,7 & 88,7 & 88,1 & 86,7 & 83,0 & 84,7 \\
\hline MISSILES & 52,1 & 43,7 & 61,1 & 56,2 & 52,2 & 71,5 & 58,0 & 51,5 & 67,3 & 56,9 & 65,0 \\
\hline AIDE ALIMENTAIRE & 50,5 & 37,6 & 42,4 & 47,1 & 41,2 & 35,7 & 38,9 & - & - & - & - \\
\hline RÉGIME POLITIQUE & 35,8 & 34,6 & 48,7 & 42,4 & 46,3 & 44,5 & 44,6 & 41,2 & 42,2 & 50,7 & 42,9 \\
\hline RELATIONS & 47,9 & 27,7 & 29,5 & 24,7 & 22,2 & 22,9 & 26,8 & 20,7 & 24,9 & 25,9 & 25,5 \\
\hline \multicolumn{12}{|l|}{ INTERCORÉENNES } \\
\hline ARME NUCLÉAIRE & 39,3 & 49,2 & 66,3 & 56,6 & 63,9 & 79,5 & 75,1 & 69,9 & 76,8 & 68,4 & \\
\hline $\begin{array}{l}\text { RETOUR DES FEMMES } \\
\text { JAPONAISES } 18\end{array}$ & 35,7 & 22,0 & 27,3 & 18,2 & 18,5 & 18,8 & 19,5 & & & & \\
\hline $\begin{array}{l}\text { NÉGOCIATIONS DE } \\
\text { NORMALISATION } 19\end{array}$ & 34,4 & 37,0 & 34,6 & 35,0 & 33,0 & 30,3 & 30,7 & 25,7 & 24,2 & 23,3 & 23,0 \\
\hline " RÈGLEMENT DU PASSÉ " & 24,6 & 21,8 & 20,7 & 16,9 & 21,0 & 18,6 & 18,5 & - & - & - & - \\
\hline RÉFUGIÉS* / TRANSFUGES $*$ & 17,4 & $17,0^{*}$ & $24,9^{*}$ & $21,9 *$ & $16,9^{*}$ & $20,7^{*}$ & $18,7^{*}$ & $44,4^{* *}$ & $35,2 * *$ & $40,6^{* *}$ & $50,5^{* *}$ \\
\hline ÉCHANGES ÉCONOMIQUES & 18,6 & 8,5 & 8,8 & 8,5 & 9,2 & 11,7 & 11,6 & & & & \\
\hline ÉCHANGES CULTURELS & 9,7 & 8,0 & 6,1 & 5,2 & 6,2 & 6,3 & 6,5 & $\{11,2$ & $\{1,9$ & $\{13,8$ & $\{12,9$ \\
\hline ÉCHANGES SPORTIFS & 9,0 & 7,4 & 5,9 & 4,9 & 7,6 & 7,3 & 6,1 & & & & \\
\hline TOURISME & 8,1 & 5,2 & 5,2 & 4,5 & 5,2 & 5,4 & 4,0 & - & - & - & - \\
\hline EMBARCATIONS SUSPECTES & - & 49,2 & 43,1 & 58,7 & 38,7 & 43,8 & 36,7 & - & - & - & - \\
\hline $\begin{array}{l}\text { TRAFICS ILLÉGAUX } \\
\text { (DROGUE. ETC.) }\end{array}$ & - & - & 45,0 & 36,5 & 36,3 & 46,0 & 44,1 & - & - & - & - \\
\hline
\end{tabular}

17 Même constat pour l'intérêt que suscite le régime politique nord-coréen qui augmente, dans un contexte de passation de pouvoir de Kim Chŏng-il à son fils Kim Chŏng-ǔn 김 정 은 金正恩, de 42,2 \% en 2009 à 50,7 \% en 2010. Quant aux enlèvements de Japonais par des agents nord-coréens, la persistance d'un intérêt très élevé est le fruit d'une médiatisation massive depuis 2002 et d'une mobilisation tous azimuts des associations œuvrant pour le retour des personnes enlevées (pétitions, campagnes de boycottage des produits nord-coréens, participation aux émissions télévisées, publications, colloques, etc.) qui ont acquis avec le temps une réelle capacité d'influence au sein de la sphère publique japonaise ${ }^{19}$.

Comment expliquer un si grand écart d'intérêt entre ces différentes questions? Il nous faut d'emblée évoquer trois pistes de réflexion qui nécessiteraient un travail à part entière; on se contentera donc ici de les évoquer. La première a trait à l'état des connaissances sur la période en question et à leur diffusion; elle renvoie à la place qui est accordée à la colonisation dans la recherche universitaire et dans l'enseignement. La deuxième touche à la notion de responsabilité collective (le sentiment de responsabilité de la nation dans son ensemble pour les faits commis) et transgénérationnelle (se sentir responsable aujourd'hui pour des faits commis hier par une génération différente de celle à laquelle on appartient). Elle pourrait être résumée par le questionnement suivant: les Japonais aujourd'hui se sentent-ils responsables de la domination coloniale exercée dans un passé qu'ils n'ont pas vécu? Le troisième élément - peut-être le plus malaisé à appréhender - concerne l'existence (ou l'absence) d'un tabou entourant le passé colonial. À côté de ces trois éléments, il est possible de mettre en avant deux autres hypothèses - dont nous tenterons ici de rendre compte permettant de comprendre pourquoi le passé ne suscite qu'un intérêt limité. Il s'agit d'une part de l'apparition de thématiques concurrentes au sein de l'espace public, et d'autre part de l'existence d'un discours excluant la Corée du Nord de la normalité.

\section{L'émergence de problèmes concurrents}

19 À partir des années 1990, les relations nippo-nord-coréennes se cristallisent autour de deux foyers de tensions. Le premier est lié au développement de l'arme nucléaire (première crise de 1993-1994 suivie d'une seconde à partir de 2002) et d'un programme balistique (tirs de missiles à courte portée en direction de la mer du Japon et, surtout, tir d'un missile Taepodong-1 en août 1998 qui survola le territoire japonais). Le second 
point de tensions mérite que l'on s'y attarde un instant tant il occupe une place écrasante au sein de la société japonaise aujourd'hui. Il concerne les enlèvements de ressortissants japonais perpétrés dans les années 1970 et 1980 par des agents nordcoréens, principalement dans le but d'usurper leur identité et de se procurer des personnes capables d'enseigner la langue et les coutumes japonaises.

C'est le quotidien conservateur Sankei qui aborda en premier la question dans un article daté du 7 janvier 1980. Le journaliste se demandait alors si la disparation de trois jeunes couples dans des départements différents à l'été 1978 ainsi que la tentative avortée d'enlèvement d'un quatrième couple pendant la même période n'étaient pas le fait des services spéciaux d'une puissance étrangère. $\mathrm{Ni}$ cet article, ni ceux publiés dans l'Asahi et le Mainichi du 28 juin 1985, après que les services secrets sud-coréens ont annoncé qu'un espion nord-coréen arrêté avait avoué avoir enlevé un Japonais, n'eurent un grand retentissement dans le pays. En mai 1991, alors que le Japon et la Corée du Nord s'étaient engagés dans des négociations pour normaliser leurs relations diplomatiques, la police du département de Saitama annonça que Yi Ŭn-hye 이은혜 李恩惠 - la personne qui avait enseigné le japonais à Kim Hyŏn-hǔi ${ }^{20}$ 김현희 金賢姫 - se révélait être Taguchi Yaeko 田口八重子 ${ }^{21}$, une Japonaise disparue en 1978. Lors de la troisième session des pourparlers nippo-nord-coréens qui débuta le 20 mai suivant à Pékin, la partie japonaise aborda la question, déclenchant la colère et l'indignation de la délégation nord-coréenne qui nia l'existence de telles pratiques.

21 Le problème des enlèvements connut un tournant important en 1997 lorsque le directeur de l'Institut de recherche sur la Corée moderne, Satō Katsumi 佐藤勝已, publia dans la revue Gendai Koria (La Corée d'aujourd'hui 現代コリア) un article dans lequel il affirmait que Yokota Megumi 横田めぐみ, une jeune fille disparue à Niigata en 1977, avait bel et bien été enlevée par des agents nord-coréens. C'était la première fois que l'on mentionnait le vrai nom d'une victime d'un enlèvement. Le 2 février, le Sankei et la revue Aera relayèrent l'information et le lendemain un journaliste japonais rencontra un agent passé au Sud qui raconta avoir vu la jeune fille à P'yŏngyang. Ce témoignage, diffusé à la télévision, entraîna une vague d'émotion au sein de la population japonaise. Les choses s'accélérèrent : le 25 mars fut créé le Comité de liaison des familles des victimes enlevées par la Corée du Nord ${ }^{22}$ avec à sa tête le père de Yokota Megumi. Un mois plus tard, la Ligue parlementaire pour le secours des Japonais suspectés d'avoir été enlevés par la Corée du Nord (Kitachōsen rachi giwaku nihonjin kyūen giin renmei 北朝鮮拉致疑惑日本人救援議員連盟) fut constituée. Une liste officielle de huit cas d'enlèvement pour onze disparus fut établie en mars $2002^{23}$.

Autrefois peu relayée dans la presse, la question des enlèvements bénéficia d'une couverture médiatique sans précédent après la rencontre au sommet Koizumi-Kim du 17 septembre 2002 durant laquelle le dirigeant nord-coréen reconnut l'implication de la Corée du Nord dans les enlèvements. L'étude des éditoriaux de quatre grands quotidiens japonais - le Yomiuri Shinbun, l'Asahi Shinbun, Mainichi Shinbun et le Sankei Shinbun - datés du 18 septembre 2002, soit le lendemain de la rencontre au sommet, montre que, s'il existe incontestablement des singularités entre ces quatre journaux ${ }^{24}$, tous ont en commun le fait d'avoir relayé au second plan la question coloniale. Trois thèmes avaient dominé la rencontre au sommet: les enlèvements, les questions de sécurité et le passé colonial. Or, dans le seul éditorial de l'Asahi, le mot "domination coloniale» (shokuminchi shihai 植民地支配) n'apparaît qu'une fois lorsque le mot 《enlèvement » (rachi 拉致) apparait, lui, quatorze fois et le mot «nucléaire » (kaku 核) 
trois fois. La même tendance est observée dans des proportions similaires pour les trois autres quotidiens. En outre, l'Asahi, le Mainichi et le Sankei se contentèrent d'un compterendu factuel («le Japon a exprimé ses excuses", "un accord sur une coopération économique a été trouvé »), sans plus d'analyse ni de mise en perspective. Les éditoriaux ne remplirent pas la mission qui leur est traditionnellement dévolue: exprimer le point de vue du journal. Seul le Yomiuri jugea opportun de revenir sur la forme adoptée pour régler la question coloniale, voyant dans la «coopération économique » - et non pas les «indemnités » comme l'exigeait P'yŏngyang - une victoire diplomatique du Japon.

Le passé ne méritait-il pas un traitement plus approfondi? Certes, le format de l'éditorial exige la concision (d'autant plus qu'au Japon ce sont habituellement deux évènements différents qui sont traités dans un même éditorial). Mais sans aller jusqu'à parler de «faits divers faisant diversion » (Pierre Bourdieu), on ne peut que constater un déséquilibre - dans les éditoriaux, mais aussi dans les autres pages des quotidiens, déséquilibre encore plus manifeste dans les hebdomadaires - entre d'une part les enlèvements relayés abondamment et à travers des expressions suscitant souvent la compassion du lecteur ${ }^{25}$, et d'autre part le traitement expéditif du passé colonial.

\section{Un pays « anormal »}

La Corée fut avant et pendant sa colonisation l'objet d'un certain nombre de discours. S'il est impossible d'en faire ici une archéologie exhaustive, on peut, en revanche, rappeler les travaux de Lionel Babicz qui montrent que des «visions sur la Corée » se développèrent à l'ère Meiji (1868-1912), et qu'en dépit de «la richesse et [de] la complexité de la réflexion concernant la Corée, [il est possible d'esquisser] quelques grands axes ${ }^{26}$ qu'il définit au nombre de trois : stratégique, civilisationnel et racial ${ }^{27}$. Le premier mythe "stratégique ", perceptible dès la fin de l'époque d'Edo (1603-1868) et ayant acquis sous la plume du Premier ministre Yamagata Aritomo 山縣有朋 un statut de "quasi-dogme officiel », fait de la Corée la première ligne de défense face à la Russie et à la Chine. Le deuxième mythe, "civilisationnel », opposa une Corée barbare et retardée à un Japon civilisé et avancé. Il prit deux formes opposées : premièrement une mission civilisatrice que le Japon se devait de mener à bien, et deuxièmement la nécessité pour le Japon de «sortir de l'Asie » (datsua 脱亜) et, par là, d'abandonner la Corée à son sort. Enfin, le troisième mythe dit « racial » soulignait, lui, les similitudes entre les peuples coréen et japonais et servit comme argument pour justifier l'annexion de la Corée en 1910.

Dans la continuité de ces différents discours s'inscrit celui qui, à partir des années 1990, fit de la Corée du Nord un pays anormal. Il ne s'agit pas ici de discuter de son bienfondé, mais d'en examiner plutôt la manifestation, dont le paroxysme se situe après la reconnaissance, en septembre 2002, par Kim Chŏng-il, des enlèvements de Japonais. La presse consacra alors son énergie à décrire la Corée du Nord comme une entité en dehors de la normalitée ${ }^{28}$. Elle fut dépeinte dans les éditoriaux des grands quotidiens comme un «État inhumain ( (hiningenteki na kokka 非人間的な国家) pour le Sankei, «ni plus ni moins un État terroriste» (tero kokka sono mono テロ国家そのもの) pour le Yomiuri. L'Asahi, blâmé un temps pour ses positions jugées trop favorables à l'égard du Nord, fut tout aussi critique en faisant référence à un " pays dangereux » (kiken na kuni 危険な国), un « pays anormal » (ijō na kuni 異常な国) ou encore le « pays des hors-la- 
loi » (muhōsha no kuni 無法者の国). Le Mainichi parla, lui, d'«État conspirateur » (bōryaku kokka 謀略国家). Dans un article du 18 septembre 2002, le chef du service politique du Mainichi écrivait qu'« il [était] de la responsabilité du Japon, voisin de la Corée du Nord, de guider ce pays vers la normalité ». Même constat dans l'éditorial de l'Asahi daté du 20 septembre: "le gouvernement japonais doit prendre en compte le fait que la Corée du Nord est un pays anormal et faire en sorte, en entamant des négociations, qu'il évolue vers la normalité ". Cette description ne se limita pas à la presse écrite. Takasaki Sōji, historien et spécialiste des relations nippo-coréennes, témoigne de l'ambiance médiatique post-17 septembre :

À partir du mois de décembre [2002], la campagne anti-Corée du Nord prit une ampleur sans précédent dans les hebdomadaires, dans les publications et à la télévision [...]. Les émissions populaires diffusèrent sans relâche, parfois jusqu'à quatre fois par jour - le matin, le midi, en début et fin de soirée - des témoignages facilement recueillis auprès de transfuges nord-coréens. Ces derniers racontaient les difficultés auxquelles ils avaient été confrontés, la faim, les enfants qui mendient, les conditions de vie misérables dans les camps de concentration. Pour distraire les téléspectateurs, on diffusa également une compilation d'émissions nord-coréennes montrant des parades militaires, des jeunes femmes plantureuses dansantes, le spectacle des enfants au sourire forcé, le tout commenté par un présentateur nord-coréen à la voix grandiloquente ${ }^{29}$.

Un coup d'œil sur les ouvrages publiés à la même époque révèle une tendance similaire, la Corée du Nord y étant présentée tantôt comme un régime cruel et débauché, tantôt comme un cabinet de curiosités ${ }^{30}$. Cette vision, qui emprunte des supports multiples du brûlot au manga en passant par l'Internet -, rend difficile une réflexion ou prise de parole dépassionnée sur le pays. Quant au passé, revenir sur des faits anciens n'apparaîtrait-il pas inopportun alors qu'un monstre menace le présent ?

Les liens personnels qu'entretenaient certains hommes politiques japonais de premier plan avec P'yŏngyang, le rapprochement Nord-Sud et l'effondrement du bloc socialiste conduisirent à la tenue de pourparlers entre le Japon et la Corée du Nord à partir du début des années 1990. Durant ces négociations qui visaient à normaliser les relations diplomatiques entre les deux États, les modalités du "règlement du passé » colonial furent âprement débattues jusqu'à ce qu'un consensus soit trouvé avec la signature de la Déclaration de P'yŏngyang le 17 septembre 2002. Cette Déclaration fait en effet mention d'excuses pour la domination coloniale et prévoit la mise en œuvre d'une "coopération économique ", dénomination pudique de ce qui constitue de facto le versement d'indemnités pécuniaires et rappelle la manière adoptée pour la Corée du Sud plus de trente-cinq années auparavant. Cet accord ne permit pourtant pas la normalisation des relations nippo-nord-coréennes. Ceci s'explique par l'existence de problèmes sécuritaires qui revêtent une acuité particulière depuis cette dernière décennie : développement d'armes balistiques et nucléaires, mais aussi - et surtout peut-être - la non-résolution de la question des enlèvements de Japonais que la Corée du Nord, par la voix de son dirigeant, reconnut à l'occasion de la rencontre au sommet du 17 septembre 2002. La persistance de ces problèmes sécuritaires (abondamment médiatisés) et l'idée corollaire d'un «État voyou » dominant les discours sur le Nord 
produit un oubli japonais de la domination coloniale (pourtant largement étudiée), domination coloniale que le régime de P'yŏngyang instrumentalise depuis sa fondation pour légitimer la place de Kim Il-sŏng et de sa descendance au sommet du pouvoir.

Au-delà des mots apparaît le risque d'amalgames et de dérives dont le discours excluant la Corée du Nord de la normalité est porteur. N'est-ce pas ce dernier qui conduisit à des actes de violence sur la communauté coréenne au Japon, relique du passé colonial scindée en deux par la guerre froide ${ }^{31}$ ? Le journaliste Yamaguchi Masanori avance le nombre de 291 cas d'actes vexatoires ou de menaces à l'encontre des établissements scolaires nord-coréens ${ }^{32}$ ou de leurs élèves pour la période allant du $17 \mathrm{au}$ 24 septembre 2002, soit juste après la visite de Koizumi Jun.ichirō à P'yŏngyang ${ }^{33}$. Le 17 septembre, par exemple, quelqu'un téléphona à l'école primaire coréenne d'ōsaka et menaça de "tuer des élèves ». Le jour suivant, on pouvait lire le commentaire «je vais vous kidnapper » laissé sur le site Internet d'un établissement scolaire du Tōhoku. Le même jour, un homme donna un coup de pied à une fillette dans le bus qui la conduisait à son école dans la ville de Yokohama. Cet établissement reçut un appel intimant l'ordre aux Coréens de « rentrez [chez eux] en Corée ». Ce fut comme s'il n'y avait plus de différences entre le tyran et le peuple qu'il oppresse. Comme si stigmatiser la population coréenne au Japon permettrait, sans doute, de corriger cette anormalité, de combattre ce mal lointain. Les péchés du passé ne pourraient-ils pas d'ailleurs s'absoudre dans cette lutte?

\section{NOTES}

1. Il s'agit du traité d'amitié nippo-coréen (nitchō shūkō jōki 日朝修好条規) ou traité de Kanghwa (kōkatō jōyaku 江華島条約) conclu en 1876.

2. Citons notamment la tentative d'assassinat du président sud-coréen par des agents nordcoréens introduits dans le palais présidentiel, en janvier 1968, et dans le Théâtre national, en août 1974. En lien direct avec le Japon : l'accueil des membres de l'Armée rouge japonaise (Nihon sekigun 日本赤軍) en Corée du Nord après que ces derniers ont détourné, en mars 1970, un Boeing de la Japan Airlines. En octobre 1983 un attentat à la bombe visa le président sud-coréen Chŏn Tu-hwan, alors en visite officielle en Birmanie. Si ce dernier échappa à l'attentat, son Ministre des affaires étrangères ainsi que d'autres officiels furent tués. Cet acte de terrorisme conduisit à la rupture des relations diplomatiques entre P'yŏngyang et Rangoon jusqu'en 2007. Enfin, en novembre 1987, soit quelques mois avant l'ouverture des Jeux olympique d'été à Séoul, l'explosion en vol d'un appareil de la compagnie sud-coréenne Korean Air, parti de Bagdad pour Séoul. La totalité des passagers et du personnel navigant périrent. Deux agents nord-coréens descendus lors de son escale à Abu Dhabi furent arrêtés; un des deux agents parvint à s'empoisonner alors que l'autre, une femme du nom de Kim Hyŏn-hǔi, fut transférée en Corée du Sud et reconnut sa responsabilité dans l'attentat.

3. Pour une réflexion sur les excuses étatiques, voir par exemple Jean-Cassien Billier, « Le paradoxe des excuses ", in Raison publique, $\mathrm{n}^{\circ} 10,2009$, p. 139-156.

4. Der Spiegel, qui fit sa une avec la photographie du chancelier agenouillé traversée par un bandeau portant l'interrogation «Brandt devait-il s'agenouiller?» (Durfte Brandt knien?), publia 
un sondage selon lequel $48 \%$ des personnes interrogées trouvaient ce geste "exagéré» (übertrieben), contre $41 \%$ qui le considéraient « approprié » (angemessen).

5. L'intitulé officiel de ce texte, qui a pris la forme d'une décision du Cabinet (kakugi kettei 閣議決 定) engageant à ce titre l'ensemble des membres le composant, est: «Murayama naikaku sôridaijin danwa ‘Sengo gojisshûnen no shûsen kinenbi ni atatte’” 村山内閣総理大臣談話「戦 後 50 周年の終戦記念日にあたって」(Convesation du Premier ministre Murayama «À l'occasion du cinquantième anniversaire de la fin de la guerre »). Les textes officiels et accords internationaux mentionnés dans le présent article sont issus de la base de données «Sekai to Nihon»世界と日本 (Le Japon et le monde) du site Internet de l'Institut de la culture orientale (Tōyō bunka kenkyūjo 東洋文化研究所) de l'Université de Tōkyō.

6. Il convient de ne pas négliger la portée «interne" de la démarche. Alors que le monde soviétique vivait ses dernières heures, les relations nippo-nord-coréennes restaient, avec les «territoires du Nord» (hoppō ryōdo 北方領土) - revendiqués à la fois par Tōkyō et Moscou - et quelques autres conflits territoriaux, les derniers foyers de tensions qui préoccupaient politiciens et diplomates japonais. Aussi, en cas de succès, la normalisation des relations entre le Japon et la RPDC aurait permis à Takeshita de laisser son nom dans l'histoire - comme le fit en son temps Tanaka Kakuei avec la Chine populaire -, peut-être même de briguer un nouveau mandat.

7. Le Parti libéral-démocrate, le Parti socialiste du Japon et le Parti du travail de Corée.

8. Voir l'étude d'Ōtake Hideo 大获秀夫, Koizumi Jun.ichirō popyurizumu no kenkyū 小泉純一郎ポ ピュリズムの研究 (Étude sur le populisme de Koizumi Jun.ichirō), Tōkyō, Tōyōkeizai 東洋経済, 2006, 270 p., p. 204.

9. À savoir les "questions fondamentales" (notamment les excuses pour la domination coloniale), des «questions économiques» (notamment les réparations et les droits de réclamation), les «questions internationales " (notamment le nucléaire) et enfin les "questions diverses» (notamment le statut juridique des Coréens résidant au Japon ainsi que le sort des femmes japonaises parties vivre en RPDC avec leurs maris coréens).

10. Notons que le texte japonais utilise owabi お詫び, terme vernaculaire, alors que le texte coréen utilise, lui, sajoe 사죄 qui a un équivalent à la fois sémantique et graphique en japonais, shazai 謝罪.

11. À l'époque, les deux parties, conscientes qu'il serait difficile d'apporter la preuve de chaque spoliation et d'estimer la valeur de chaque bien spolié, se mirent d'accord sur un règlement forfaitaire. Signé en 1965 avec le traité général, l'accord relatif à la solution des problèmes concernant les biens, les droits de réclamations et la coopération économique prévoyait que le Japon versât à la République de Corée une somme forfaitaire de trois cents millions de dollars américains et lui accordât des prêts à long terme et à intérêt réduit d'un montant total de deux cents millions de dollars. En plus de cette somme, une note échangée entre les deux gouvernements engageait le Japon à octroyer des crédits commerciaux privés à la République de Corée et/ou à ses ressortissants pour un montant dépassant les trois cents millions de dollars. Pour un commentaire en français du traité nippo-sud-coréen, voir Lazar Focsaneanu, «Les Relations nippo-coréennes et les traités de Tokio du 22 juin 1965 », in Politique étrangère, vol. 30, $\mathrm{n}^{\circ} 4,1965,470$ p., p. 369-409.

12. Tanaka Hitoshi 田中均 \& Tahara Sōichirō 田原総一郎, Kokka to gaikō 国家と外交 (L'État et la diplomatie), Tōkyō, Kōdansha 講談社, 2005, 268 p., p. 49.

13. Voir par exemple en français, l'Histoire abrégée de l'activité révolutionnaire du camarade Kim Il Sung publiée par l'Institut de recherche sur l'histoire du Parti du Comité central du Parti du travail de Corée aux Éditions en langues étrangères en 1969.

14. George Orwell, 1984, Gallimard, 1950, 376 p., p. 23. Traduction française d'Amélie Audiberti.

15. Certains auteurs, à l'image de Narushige Michishita dans son North Korea's Military-Diplomatic Campaigns 1966-2008, London, Routledge, 2009, 295 p., considèrent l'acquisition d'une aide économique et la survie du régime (les deux sont en réalité liés) comme les objectifs premiers de 
la RPDC depuis les années 1990, expliquant ainsi la volonté de P’yŏngyang de normaliser ses relations diplomatiques avec Tōkyō.

16. Voir, par exemple, Ōe Shinobu 大江志乃部夫 et. al., Kindai nihon to shokuminchi 近代日本と植 民地 (Le Japon moderne et ses colonies), Iwanami shoten 岩波書店, huit volumes publiés entre 1992 et 1993.

17. Ce choix de réponse n'est plus proposé aux sondés dans les enquêtes conduites en 2008 et 2010. Si des interrogations demeurent quant à la raison de cette absence, sa place dans les choix possibles proposés jusqu'en 2007 tend à prouver que, pour les concepteurs du sondage, l'expression "règlement du passé » n'était pas à comprendre comme à penser exclusivement dans le cadre interétatique. Dans le cas contraire, le Japon et la Corée du Nord ayant trouvé un consensus sur la question exprimé dans la déclaration de P'yŏngyang de septembre 2002, il n'aurait pas été logique de proposer ce choix après cette date.

18. Sondages effectués chaque année au mois d'octobre auprès d'une population de 3000 personnes (avec un nombre de réponses valables variant de 1700 à 2100 en fonction des années) âgées de vingt ans ou plus sur l'ensemble du territoire japonais. Source: Bureau du gouvernement (unité : \%).

19. Donnons juste un exemple récent. Alors que les élections générales d'août 2009 consacrèrent la victoire du Parti démocrate (PDJ) - mettant fin au règne quasi interrompu du PLD depuis 1955 -, une rumeur circula selon laquelle Tanaka Hitoshi, l'architecte de la rencontre du 17 septembre 2002 qui avait quitté le ministère des Affaires étrangères en 2005, pourrait se voir confier des fonctions au sein du nouveau gouvernement. L'Association pour le secours des Japonais enlevés par la Corée du Nord (Sukuu-kai 救う会) et l'Association des familles de victimes enlevées par la Corée du Nord (Kazoku-kai 家族会), les deux principales associations œuvrant pour le retour des Japonais kidnappés, réagirent le 4 septembre 2009 en publiant un communiqué conjoint dans lequel elles se disaient «fermement opposés à toute participation, sous quelque forme que ce soit, dans le nouveau gouvernement et dans son processus décisionnel, de $\mathrm{M}$. Tanaka ». Ce dernier reste mal vu aux yeux de ces associations car il avait promis à son interlocuteur nord-coréen que les cinq Japonais enlevés rentrés "temporairement » au Japon quelques temps après la rencontre de septembre 2002 retourneraient en Corée du Nord. Que la rumeur fût fondée ou non, Tanaka Hitoshi ne participa pas au gouvernement. En revanche, le nouveau Premier Ministre Hatoyama Yukio 鳩山由紀夫 ne manqua pas de faire explicitement référence aux enlèvements lors de son discours devant l'Assemblée générale des Nations Unies, le 24 septembre 2009.

20. Voir la deuxième note de bas de page du présent article.

21. La police ne donna alors que les initiales «T. Y. » et publia un portrait robot.

22. Kitachōsen ni yoru rachi higaisha kazoku renraku-kai 北朝鮮による拉致被害者家族連絡会, souvent abrégé en «Association des familles » (kazoku-kai 家族会).

23. En mars 1988, le directeur de la Commission nationale de la Sûreté publique, Kajiyama Seiroku 梶山静六, reconnut devant la Commission du Budget du Sénat l'implication de la Corée $\mathrm{du}$ Nord dans les enlèvements. Le nombre d'enlèvements reconnus officiellement par le gouvernement japonais a évolué avec le temps. Mai 1997 : sept affaires d'enlèvements pour dix personnes enlevées; mai 2002 : huit affaires pour onze enlevés; octobre 2002 : dix affaires pour quinze enlevés ; avril 2005 : onze affaires pour seize enlevés ; novembre 2006 : douze affaires pour dix-sept enlevés.

24. L'Asahi, par exemple, adopte une position relativement souple vis-à-vis de P'yŏngyang en appelant le gouvernement japonais à poursuivre le dialogue et à ne pas infliger de sanctions économiques au régime. Le Mainichi est le seul des quatre quotidiens à faire remarquer - à juste titre - que la reconnaissance des enlèvements et les excuses de Kim Chŏng-il ne figurent sur aucun document écrit officiel révélé au public. Le Sankei souligne, lui, la contradiction qui existe 
entre le statut tout-puissant du Secrétaire général du Parti du Travail de Corée et le fait que, selon ses dires, il n'était pas au courant des évènements.

25. L'Asahi intitule son éditorial «Un dénouement trop triste. Négocier et normaliser pour accélérer le changement [en Corée du Nord] » (Kanashisugiru ketsumatsu. Henka o unagasu seijōka $k o ̄ s h o ̄ o$ 悲しすぎる結末 変化を促す正常化交涉を) et fait part aux lecteurs, dès la première ligne, d'un «douloureux dénouement»(itamashii ketsumatsu 痛ましい結末). Il poursuit en parlant d'actes « insupportables [...] quand on pense à l'amertume [des] familles [des victimes]» (sono kazoku no munensa o omou to itatamarenai その家族の無念さを思うと、いたたまれない) avant de se mettre à la place de ces dernières en affirmant qu'il ne fait «aucun doute que ce qu'elles endurent dépasse l'imaginable» (sōzōo zessuru taegatasa ni chigai nai 想像を絶する耐え 難さに違いない). Le Mainichi parle, lui, d'actes «tellement cruels» (amari ni mo mugoi あまりに もむごい), et affirme qu'il sera « difficile [pour le peuple japonais] de surmonter ce choc et de calmer [sa] colère» (kono shōgeki to ikari wa yōi ni norikoerarenai この衝撃と怒りは、容易に乗り 越えられない). Il se met enfin à la place des victimes : «combien elles auraient aimé rentrer chez elles, auprès de leurs proches » (donna ni kazoku no moto he kaeritakatta darō どんなに家族の もとへ帰りたかっただろう). Pour sa part, le Sankei commence son éditorial par la phrase suivante : « Le 17 septembre 2002 restera certainement dans les mémoires comme un jour où le Japon a pleuré amèrement un dénouement cruel » (amari ni mo hidoi ketsumatsu ni nihon ga dōkoku shita hi toshite heisei jūyonen kugatsu jūshichinichi wa kioku sareru dearō あまりにも酷い結末に日本 が慟哭した日として、平成十四年九月十七日は記憶されるであろう). Quelques lignes plus loin, on peut lire dans ce même éditorial que, face à ce "crime d'État", le peuple japonais « partage colère et tristesse » (ikari to kanashimi o kyōyū suru 怒りと悲しみを共有する).

26. Lionel Babicz, Le Japon face à la Corée à l'époque Meiji, Maisonneuve \& Larose, 2002, 271 p., p. 220-221.

27. En réalité, la distinction de ces trois «mythes » comme trois éléments indépendants est un peu artificielle car, comme le souligne Lionel Babicz, "les trois forces sont en action simultanément, s'agrègent et se complètent - déforment la réalité, expliquent le présent, contribuent à la mobilisation des passions, construisent ensemble autant de visions de la Corée ", ibidem, p. 221.

28. Pour une analyse détaillée, on consultera la deuxième partie de l'ouvrage compilé par le Jinken to hōdō renrakukai 人権と報道連絡会 (Comité de liaison sur les droits de l'homme et l'information) Kenshō “rachikikokusha” masukomihōdō 検証・「拉致帰 国者」マスコミ報道 (Le Traitement médiatique du retour des Japonais enlevés à l'étude), Shakai hyōronsha 社会評論 者, 2003, 261 p. Y sont passés au crible les articles des quotidiens et hebdomadaires japonais parus du 17 septembre au 12 décembre 2002, traitant de la Corée du Nord.

29. Takasaki Sōji 高崎宗司, Kenshō nitchō kōshō 検証 日朝交涉 (Les Négociations nippo-nordcoréennes à l'épreuve), Tōkyō, Heibonsha, coll. «Heibonsha shinsho » 平凡社新書, 2004, 237 p., p. 189-190.

30. Pour cet aspect, citons, entre autres, Kitachōsen no kurashi 北朝鮮の暮らし (La vie quotidienne en Corée du Nord) de Miyatsuka Toshio 宮塚利雄 (Shōgakukan, décembre 2002, 217 p.), qui est un catalogue commenté des objets du quotidien utilisés en Corée du Nord (savon, plaque d'immatriculation, préservatif, etc.); Kitachōsen toribia 北朝鮮トリビア (Curiosités sur la Corée du Nord) de Katō Masaki 加藤将輝 et Nakamori Akio 中森明夫 (Asuka shinsha, 2004, 127 p.) ; Kitachōsen no fushigi na jinmin seikatsu 北朝鮮の不思議な人民生活 (L'Étrange quotidien du peuple nord-coréen, Takarajimasha, octobre 2006, 97 p.), dont la couverture promet au lecteur de lui raconter la vie des Nord-coréens à travers " plein de photos exclusives et importantes ».

31. La communauté coréenne restée au Japon après 1945 s'est organisée, suivant la division de la péninsule coréenne, autour de deux entités : l'Union des Coréens résidant au Japon (Zainihon daikanminkoku mindan 在日本大韓民国民団; abréviation：Mindan) pro-Sud, et l'Association 
générale des Coréens résidant au Japon (Zainihon chōsenjin sōrengō-kai 在日本朝鮮人総連合会: sōren) pro-Nord.

32. Il existe plusieurs établissements scolaires nord-coréens établis sur le territoire japonais, fréquentés quasi exclusivement par les résidants coréens du Japon « affilliés » à la Corée du Nord. Regroupés sous le terme général d' «écoles coréenne» (Chôsen gakkô 朝鮮学校), ces établissements permettent une scolarisation de l'école élémentaire au lycée. Il existe également une université affiliée à la Corée du Nord.

33. Yamaguchi Masanori 山口正紀, «Rachi isshoku hōdō ga kakusu “miseisan no kako” "拉致一 色報道が隠す〈未清算の過去〉 (Un Passé qui ne passe pas: ce que cache une information exclusivement consacrée aux enlèvements) in Jinken to hōdō renrakukai, op. cit., p. 58. Yamaguchi s'appuie sur les chiffres que mentionne le numéro 1124 du 15 octobre 2002 de la revue Hantennōsei undō Panchi! 反天皇制運動パンチ! publiée par le Comité de liaison du Mouvement anti-système impérial (Han-tennōsei undō renraku-kai 反天皇制運動連絡会).

\section{RÉSUMÉS}

Le présent travail a trait à la colonisation de la Corée par le Japon à travers deux axes de réflexion: la question coloniale en tant qu'enjeu des négociations nippo-nord-coréennes après 1945, puis la place occupée depuis ces dix dernières années par cette même question au sein de la sphère publique au Japon.

Lors des négociations entamées en 1991 et qui visaient à normaliser les relations diplomatiques entre les deux États, les modalités du « règlement du passé » colonial furent âprement débattues jusqu'à ce qu'un consensus soit trouvé, avec la signature de la Déclaration de P'yŏngyang le 17 septembre 2002. Cette Déclaration fait mention d'excuses pour la domination coloniale et prévoit la mise en œuvre d'une "coopération économique ", dénomination pudique de ce qui constitue de facto le versement d'indemnités pécuniaires, ce qui rappelle la manière adoptée pour la Corée du Sud plus de trente-cinq années auparavant.

La persistance de problèmes sécuritaires abondamment médiatisés - développement d'armes balistiques et nucléaires, mais aussi la non résolution de la question des enlèvements de Japonais par la Corée du Nord - et l'idée corollaire d'un « État voyou » dominant les discours sur le Nord, produit un oubli japonais de la domination coloniale (pourtant largement étudiée), domination coloniale que le régime de P'yŏngyang instrumentalise depuis sa fondation pour légitimer la place de Kim Il-sŭng et de sa descendance au sommet du pouvoir.

The present paper is an attempt on grasping with the Japanese colonization in Korea through two main issues: the colonial past as an important stake in the Japan-North Korea negotiations since 1945, and the status of the Japanese colonization among the Japanese public debate.

In 1991, during the negotiations aiming at the normalization between Japan and North Korea, "settling the past » constituted a main issue before reaching to the consensus of the P'yŏngyang Declaration, the 17th of September 2002. The Declaration mentions apologies for the colonial domination and preconizes a strong "economical cooperation", meaning actually the payment of indemnities to North Korea, and re-enacting the same type of resolution decided toward South Korea some 35 five years ago.

In Japan, the persistence of regional security issues with North Korea (the threat of ballistic and nuclear missiles, repeated abductions of Japanese citizens) and the well mediatized idea of North 
Korea as a « rogue state » tends to obliterate, in the public Japanese mind, the colonial past and the former Japanese domination in Korea. Although very well documented et studied in Japan among scholars, the colonial domination, thus, constituted a weapon of choice in the selflegitimization rhetoric of the P'yŏngyang regime and Kim Il-sŭng, since its very foundation.

\section{INDEX}

\section{Index géographique : Corée}

Mots-clés : colonisation, diplomatie, règlement du passé, enlèvements, Koizumi Jun.ichirō, Kim Il-sŭng

Keywords : Korea, Colonization, Diplomacy, Settling the Past, Abductions, Koizumi Jun.ichirō, Kim Il-sŭng

Thèmes : histoire

Index chronologique : Shōwa (1945-1989), Heisei (1989-) 